\title{
Response to "Hyponatremia in spinal cord injury patients: new insight into differentiating between the dilution and depletion forms'
}

\author{
Spinal Cord (2015) 53, 895; doi:10.1038/sc.2015.130; published online 28 July 2015
}

Kriz et al. ${ }^{1}$ have carried out a retrospective study on Hyponatremia in 353 patients with spinal injuries between 2008 and 2012. The patients were admitted at the spinal injury centre at an average of 44.3 days after injury but 5 patients were admitted within 2 weeks of injury. They did not specify whether the patients had received an operation upon their spines, steroids, nor did they define what they meant by significant hypotension. They speculated that the changes that they found could be due to:

1. Impairment of autonomic function.

2. Reduced renal blood flow.

3. Significant hypotension.

4. Urinary tract infection which many of the patients suffered from.

5. Altered antidiuretic hormone $(\mathrm{ADH})$ secretion.

The problem of altered fluid balance following an acute spinal injury was first mentioned by Ambrose Paré ${ }^{2}$ in 1649, then by Gordon Holmes $^{3}$ in 1915 when he observed significant oliguria following a spinal injury.

In order to investigate this problem further, a prospective study was carried out at the National Spinal Injury Centre by Silver et al., ${ }^{4}$ studying patients admitted from 1965 onwards. A total of 27 water load tests were carried out on 20 patients. The test included measurement of serum osmolality to confirm absorption of 1 litre of ingested water. Impaired response to the water load was obtained in 17 tests; in 12/13 between 1 and 5 days after onset of the cord lesion and in 5/14 more than 2 weeks after injury. The blood pressure was substantially normal throughout thus discounting hypotension and we concluded that it was due to secretion of $\mathrm{ADH}$.

An earlier study in 1966 by Doggart et al. ${ }^{5}$ found that in the acute stages, glomerular filtration rates, as indicated by the creatinine clearances test, was found remarkably normal thus the impaired blood supply to the kidneys was not a factor.

In 2004, a further study was carried out to examine the effects of sodium loading on acute spinal injury patients admitted between 1962 and $1964 .{ }^{6} \mathrm{~A}$ total of 53 studies were carried out on 52 patients. In all, 49 patients were studied during the first 10 days after injury as follows: a control group of 39 patients received no supplementary sodium, two received $\mathrm{NaCl}$ infusion, $2 \mathrm{NaCl}$ tablets orally, and 6 received $\mathrm{NaCl}$ infusion followed by $\mathrm{NaCl}$ tablets orally. Four patients were studied for $>15$ days after injury ( 1 of these had been studied in the acute stage); they received $\mathrm{NaCl}$ infusion and 1 received in addition $\mathrm{NaCl}$ tablets for 4 subsequent days. In all patients urinary sodium excretion was minimal on day 2 and increased thereafter. On days 2-6, it was significantly lower in patients with a complete transection of the cervical cord than in patients with lower lesions. In the early studies, 9 patients excreted $<40 \%$ of the administered load within $24 \mathrm{~h}$. In 4 of these patients excretion was $10 \%$ or less. In the later studies, 3 of the 4 patients excreted at least $80 \%$ of the infused $\mathrm{Na}^{+}$on the same day.

These patients were not suffering from a urinary tract infection, they did not receive steroids and they did not have their spines operated on.

We concluded that the alterations in fluid balance were due to endocrine changes as a response to trauma. These were more significant in patients with a spinal cord injury.

\section{CONFLICT OF INTEREST}

The author declares no conflict of interest.

JR Silver Stoke Mandeville Hospital, Buckinghamshire, UK E-mail: jrussellsilver@btconnect.com

1 Kriz J, Schuck O, Horackova M. Hyponatremia in spinal cord injury patients: new insight into differentiating between the dilution and depletion forms. Spinal Cord 2015; 53: 291-296.

2 Paré A. The works of that famous chirurgion Ambroise Paré. Translated by T. Johnson (1649). Cotes and Dugard: London, 1702, 435-436.

3 Holmes G. Spinal injuries of warfare. The clinical symptoms of gunshot injuries of the spine. Br Med J 1915; ii: 815-821.

4 Silver JR, Doggart JR, Burr RG. The reduced urinary output after spinal cord injury: a review. Spinal Cord 1995; 33: 721-725.

5 Doggart JR, Guttmann L, Silver JR. Comparative studies on endogenous creatinine and urea clearances in paraplegics and tetraplegics. Paraplegia 1966; 3: 229-242.

6 Silver JR, Doggart JR. Reduced sodium output following acute spinal injury. Spinal Cord 2004; 42: 191-198. 